\title{
Charmonium in nuclear matter and nuclei
}

\section{J.J. Cobos Martínez, ${ }^{a}{ }^{*}$ K. Tsushima, ${ }^{b}$ G. Krein ${ }^{c}$ and A.W. Thomas ${ }^{d}$}

${ }^{a}$ Departamento de Física, Universidad de Sonora, Boulevard Luis Encinas J. y Rosales, Colonia Centro, Hermosillo, Sonora 83000, México

${ }^{b}$ Laboratório de Física Teórica e Computacional-LFTC, Universidade Cidade de São Paulo, 01506-000, São Paulo, SP, Brazil

${ }^{c}$ Instituto de Física Teórica, Universidade Estadual Paulista, Rua Dr. Bento Teobaldo Ferraz, 271 - Bloco II, 01140-070, São Paulo, SP, Brazil

${ }^{d}$ CSSM, School of Physical Sciences, University of Adelaide, Adelaide SA 5005, Australia

E-mail: jesus.cobos@fisica.uson.mx, kazuo.tsushima@gmail.com, gkrein@ift.unesp.br, anthony.thomas@adelaide.edu.au

We present results for the $\eta_{c}$-nucleus bound state energies for various nuclei using an effective Lagrangians approach. The attractive potentials for the $\eta_{c}$ in the nuclear medium originate from the medium-modified intermediate $D D^{*}$ state in the $\eta_{c}$ self energy, using the local density approximation. Our results suggest that the $\eta_{c}$ should form bound states with all the nuclei considered.

\footnotetext{
*** 10th International Workshop on Charm Physics (CHARM2020), ***

*** 31 May - 4 June, 2021 ***

*** Mexico City, Mexico - Online ***
}

\footnotetext{
${ }^{*}$ Speaker
} 


\section{Introduction}

Quantum chromodynamics (QCD) is the accepted fundamental theory of the strong interactions. However, a quantitative understanding of the strong force and strongly interacting matter in vacuum and extreme conditions of temperature and density from the underlying theory is still limited. Even though there are numerous reasons to study charmonium states, the study of the interactions of these states with atomic nuclei offers potential opportunities to gain an understanding of the workings of strongly interacting matter and QCD. Since charmonium and nucleons do not share light $(u, d)$ quarks, the OZI rule suppresses the interactions mediated by the exchange of mesons made of only light quarks. If such states are indeed bound to nuclei, it is therefore important to search for other sources of attraction which could lead to the binding of charmonia to atomic nuclei. Since the suggestion of Brodsky [1], more than three decades ago, that charmonium states may be bound with nuclei, a large amount of research looking for alternatives to the meson exchange mechanism has accumulated over the years to investigate the possible existence of such exotic states [2]. The discovery of such bound states would represent an important step forward in our understanding of the nature of strongly interacting systems.

There is a great amount of evidence that the internal structure of hadrons changes in medium and this must be taken into account when addressing charmonium in nuclei. Studies carried by some of us $[3,4]$ have shown that the effect of the nuclear mean fields on the intermedium $D \bar{D}$ state is of particular importance in the $J / \Psi$ case; the modifications induced by the strong nuclear mean fields on the $D$ mesons' light-quark content enhance the self-energy in such a way as to provide an attractive $J / \Psi$-nucleus effective potential. We extended this approach to the case of $\eta_{c}$ in Ref. [5] by considering an intermediate $D D^{*}$ state in the $\eta_{c}$ self-energy with the ligth quarks created from the vacuum. Here the effective scalar and vector meson mean fields in the nuclear medium couple to the light $u$ and $d$ quarks in the charmed mesons and this, as in the case of the $J / \Psi$, provides attraction to the $\eta_{c}$ in the nuclear medium. Recently, we have extended this approach to the case of bottomonium [6].

This paper is organized as follows. In Sec. 2 we briefly discuss the computation and present results for the mass shift of the $\eta_{c}$ in symmetric nuclear matter. Using the results of Sec. 2, together with the density profiles of the nuclei studied, in Sec. 3 we present results for the scalar $\eta_{c}$-nucleus potentials, as well as the corresponding bound state energies. Finally, in Sec. 4 we present our conclusions.

\section{2. $\eta_{c}$ in nuclear matter}

In computing the $\eta_{c}$ mass shift in nuclear matter we take into account only the intermediate $D D^{*}$ state contribution to the $\eta_{c}$ self-energy; see Refs [5, 6] and references therein for details. The effective interaction Lagrangian for the $\eta_{c} D D^{*}$ vertex is given by

$$
\mathcal{L}_{\eta_{c} D D^{*}}=i g_{\eta_{c} D D^{*}}\left[\left(\partial^{\mu} \eta_{c}\right)\left(\bar{D}_{\mu}^{*} D-\bar{D} D_{\mu}^{*}\right)-\eta_{c}\left(\bar{D}_{\mu}^{*}\left(\partial^{\mu} D\right)-\left(\partial^{\mu} \bar{D}\right) D_{\mu}^{*}\right)\right],
$$

where $D^{(*)}$ represents the $D^{(*)}$-meson field isospin doublet, and $g_{\eta_{c}} D D^{*}$ is the coupling constant. Using Eq. (1), the Feynman diagram of Fig. 1, and considering an $\eta_{c}$ at rest, the $\eta_{c}$ self-energy is 

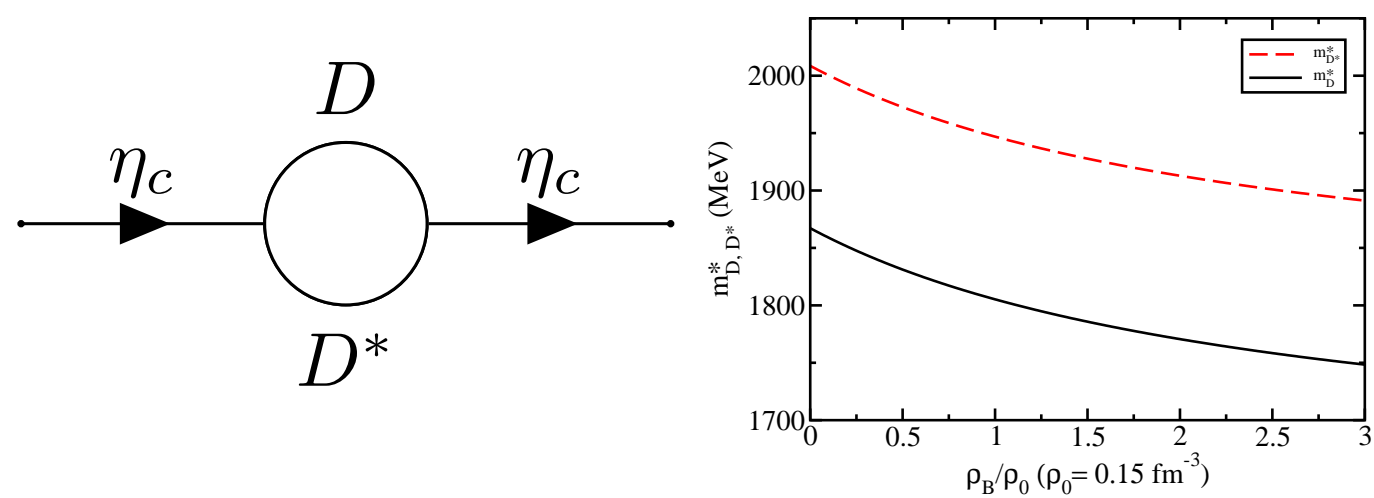

Figure 1: Left panel: Intermediate $D D^{*}$ state contribution to the $\eta_{c}$ self energy. Right panel: In-medium $D$ and $D^{*}$ meson masses in nuclear matter.

given by,

$$
\Sigma_{\eta_{c}}\left(k^{2}\right)=\frac{8 g_{\eta_{c} D D^{*}}^{2}}{\pi^{2}} \int_{0}^{\infty} \mathrm{d} k k^{2} I\left(k^{2}\right)
$$

where $I\left(k^{2}\right)$ is given by

$$
\begin{aligned}
& I\left(k^{2}\right)=\left.\frac{m_{\eta_{c}}^{2}\left(-1+k^{02} / m_{D^{*}}^{2}\right)}{\left(k^{0}+\omega_{D^{*}}\right)\left(k^{0}-\omega_{D^{*}}\right)\left(k^{0}-m_{\eta_{c}}-\omega_{D}\right)}\right|_{k^{0}=m_{\eta_{c}}-\omega_{D^{*}}} \\
& \quad+\left.\frac{m_{\eta_{c}}^{2}\left(-1+k^{02} / m_{D^{*}}^{2}\right)}{\left(k^{0}-\omega_{D^{*}}\right)\left(k^{0}-m_{\eta_{c}}+\omega_{D}\right)\left(k^{0}-m_{\eta_{c}}-\omega_{D}\right)}\right|_{k^{0}=-\omega_{D^{*}}},
\end{aligned}
$$

and $\omega_{D^{(*)}}=\left(k^{2}+m_{D^{(*)}}^{2}\right)^{1 / 2}$, with $k=|\vec{k}|$. The integral in Eq. (2) is divergent and will be regulated with a phenomenological vertex form factor, as in Refs [3-8],

$$
u_{D^{(*)}}\left(k^{2}\right)=\left(\frac{\Lambda_{D}^{2}+m_{\eta_{c}}^{2}}{\Lambda_{D}^{2}+4 \omega_{D^{(*)}}^{2}\left(k^{2}\right)}\right)^{2},
$$

with cutoff parameter $\Lambda_{D}\left(=\Lambda_{D^{*}}\right)$, by introducing the factor $u_{D}\left(k^{2}\right) u_{D^{*}}\left(k^{2}\right)$ into the integrand of Eq. (2) . The cutoff parameter $\Lambda_{D}$ is an unknown input to our calculation. Its value has been determined phenomenologically in Ref [3] to be $\Lambda_{D} \approx 2500 \mathrm{MeV}$. However, in order to quantify the uncertainity of our results to its value we present results for $\Lambda_{D}$ in the range $1500-3000 \mathrm{MeV}$.

The $\eta_{c}$ mass shift in nuclear matter is computed from the difference between the in-medium, $m_{\eta_{c}}^{*}$, and vacuum, $m_{\eta_{c}}$, masses of the $\eta_{c}$,

$$
\Delta m_{\eta_{c}}=m_{\eta_{c}}^{*}-m_{\eta_{c}}
$$

with the masses computed by solving

$$
m_{\eta_{c}}^{2}=\left(m_{\eta_{c}}^{0}\right)^{2}+\Sigma_{\eta_{c}}\left(k^{2}=m_{\eta_{c}}^{2}\right)
$$




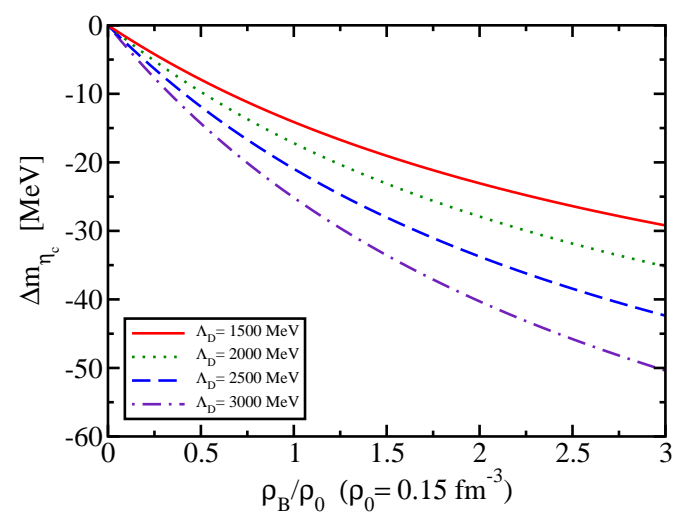

Figure 2: $\eta_{c}$ mass shift as a function of the nuclear matter density for various values of the cutoff parameter.

where $m_{\eta_{c}}^{0}$ is the bare $\eta_{c}$ mass and $\Sigma_{\eta_{c}}\left(k^{2}\right)$ is given by Eq. (2). The $\eta_{c}$ meson bare mass is fixed using Eq. (6) by fitting the physical $\eta_{c}$ mass, $m_{\eta_{c}}=2983.9 \mathrm{MeV}$. (For the other parameters in vacuum we use $g_{\eta_{c} D D^{*}}=(0.6 / \sqrt{2}) g_{J / \Psi D D}=3.24[9,10], m_{D}=1867.2 \mathrm{MeV}$, and $m_{D^{*}}=2008.6$ $\mathrm{MeV}$; see Ref. [5] for details). The $\eta_{c}$ mass in the nuclear medium is similarly computed from Eq. (6) with the self-energy calculated with the medium-modified $D$ and $D^{*}$ masses. The nuclear density dependence of the $\eta_{c}$ mass originates from the interactions of the intermediate $D D^{*}$ state with the nuclear medium through their medium-modified masses, $m_{D}^{*}$ and $m_{D^{*}}^{*}$, respectively. These in-medium masses are calculated within the quark-meson coupling (QMC) model [3, 4], in which effective scalar and vector meson mean fields couple to the light quarks in the charmed mesons [3,4]. The resulting medium-modified masses for the $D$ and $D^{*}$ mesons are shown in the left panel of Fig. 1 as a function of $\rho_{B} / \rho_{0}$, where $\rho_{B}$ is the baryon density of nuclear matter and $\rho_{0}=0.15 \mathrm{fm}^{-3}$ is the saturation density of symmetric nuclear matter. As can be seen from Fig. 1 the masses of the $D$ and $D^{*}$ mesons are reduced in the nuclear medium. In Fig. 2, we present the $\eta_{c}$ mass shift, $\Delta m_{\eta_{c}}$, as a function of $\rho_{B} / \rho_{0}$ for several values of $\Lambda_{D}$. As can be seen from Fig. 2, the effect of the nuclear medium is to shift the $\eta_{c}$ mass downwards. This happens for all values of $\Lambda_{D}$.

\section{3. $\eta_{c}$ in nuclei}

A negative mass shift for the $\eta_{c}$ means that the nuclear medium provides attraction to the $\eta_{c}$ and opens the possibility for the binding of $\eta_{c}$ mesons to nuclei. Thus, we now consider the binding of the $\eta_{c}$ to nuclei when the $\eta_{c}$ is produced inside a nucleus $A$ with baryon density distribution $\rho_{B}^{A}(r)$; see Ref. [5] for a more detailed discussion. Here we only consider the nuclei ${ }^{4} \mathrm{He},{ }^{12} \mathrm{C}$, ${ }^{16} \mathrm{O},{ }^{197} \mathrm{Au}$, and ${ }^{208} \mathrm{~Pb}$, whose baryon density distributions were also calculated within the QMC model [5], except that for ${ }^{4} \mathrm{He}$ [11]. Using a local density approximation, the $\eta_{c}$-meson potential inside nucleus $A$ is given by

$$
V_{\eta_{c} A}(r)=\Delta m_{\eta_{c}}\left(\rho_{B}^{A}(r)\right),
$$

where $\Delta m_{\eta_{c}}$ mass shift shown in Fig. 2, and $r$ is the distance from the center of the nucleus. In Fig. 3 we present the $\eta_{c}$ potentials for the nuclei mentioned above and various values of $\Lambda_{D}$. From Fig. 3 

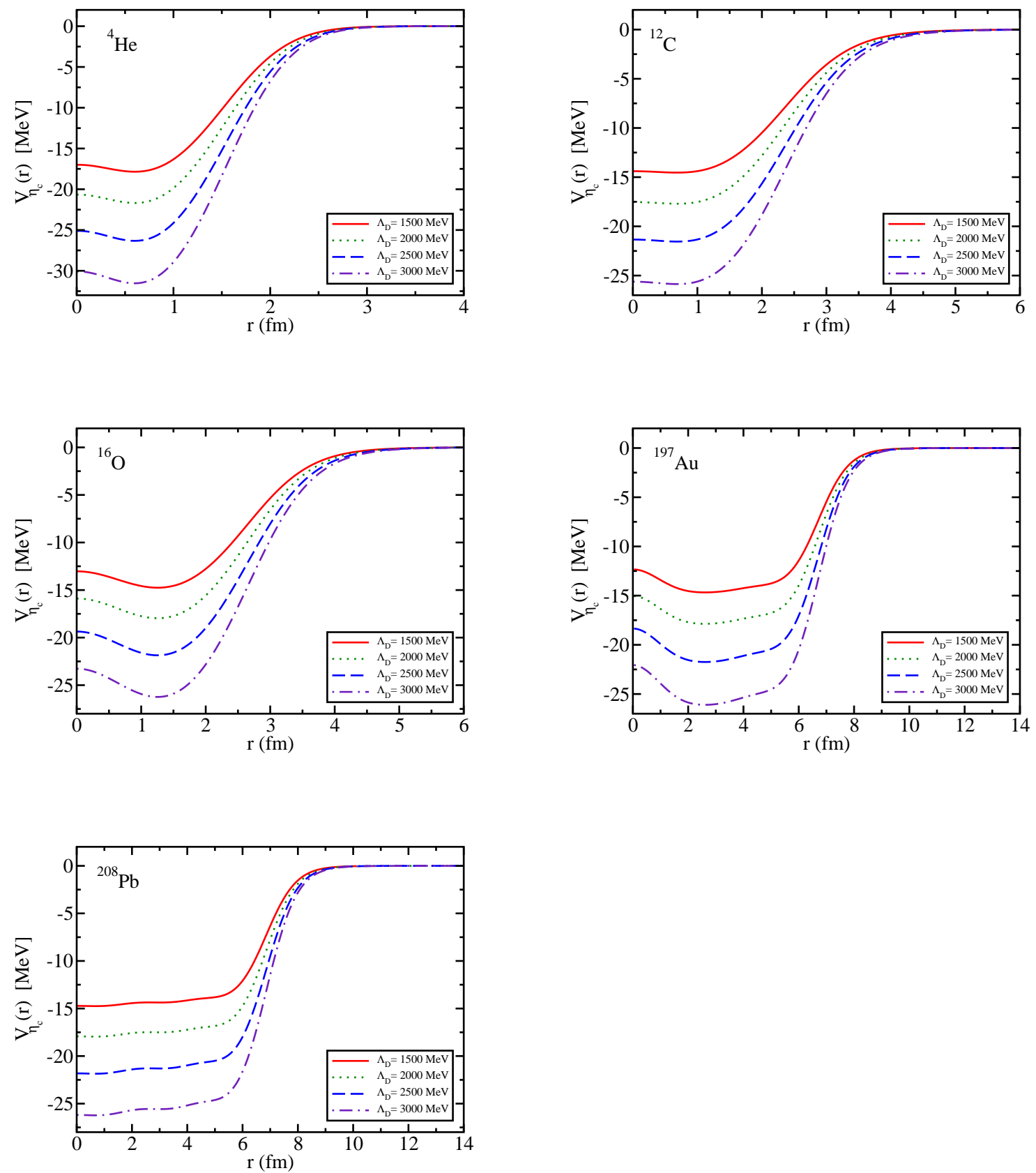

Figure 3: $\eta_{c}$-nucleus potentials for various nuclei and values of the cutoff parameter $\Lambda_{D}$. 
one can see that all $\eta_{c}$ potentials are attractive but their depths depend on $\Lambda_{D}$, being deeper for $\operatorname{larger} \Lambda_{D}$.

\begin{tabular}{ll|r|r|r|r}
\hline \hline & & \multicolumn{4}{|c}{ Bound state energies } \\
\hline & $n \ell$ & $\Lambda_{D}=1500$ & $\Lambda_{D}=2000$ & $\Lambda_{D}=2500$ & $\Lambda_{D}=3000$ \\
\hline${ }_{\eta_{c}} \mathrm{He}$ & $1 \mathrm{~s}$ & -1.49 & -3.11 & -5.49 & -8.55 \\
\hline${ }_{\eta_{c}} \mathrm{C}$ & $1 \mathrm{~s}$ & -5.91 & -8.27 & -11.28 & -14.79 \\
& $1 \mathrm{p}$ & -0.28 & -1.63 & -3.69 & -6.33 \\
\hline${ }_{\eta_{c}}^{16} \mathrm{O}$ & $1 \mathrm{~s}$ & -7.35 & -9.92 & -13.15 & -16.87 \\
& $1 \mathrm{p}$ & -1.94 & -3.87 & -6.48 & -9.63 \\
\hline${ }_{\eta_{c}}^{197} \mathrm{Au}$ & $1 \mathrm{~s}$ & -12.57 & -15.59 & -19.26 & -23.41 \\
& $1 \mathrm{p}$ & -11.17 & -14.14 & -17.77 & -21.87 \\
& $1 \mathrm{~d}$ & -9.42 & -12.31 & -15.87 & -19.90 \\
& $2 \mathrm{~s}$ & -8.69 & -11.53 & -15.04 & -19.02 \\
& $1 \mathrm{f}$ & -7.39 & -10.19 & -13.70 & -17.61 \\
\hline${ }^{208} \mathrm{~Pb}$ & $1 \mathrm{~s}$ & -12.99 & -16.09 & -19.82 & -24.12 \\
& $1 \mathrm{p}$ & -11.60 & -14.64 & -18.37 & -22.59 \\
& $1 \mathrm{~d}$ & -9.86 & -12.83 & -16.49 & -20.63 \\
& $2 \mathrm{~s}$ & -9.16 & -12.09 & -15.70 & -19.80 \\
& $1 \mathrm{f}$ & -7.85 & -10.74 & -14.30 & -18.37 \\
\hline \hline
\end{tabular}

Table 1: $\eta_{c}$-nucleus bound state energies for various nuclei. All dimensionful quantities are in $\mathrm{MeV}$.

Now we calculate the $\eta_{c}$-nucleus bound state energies for the nuclei listed above by solving the Klein-Gordon equation

$$
\left(-\nabla^{2}+m^{2}+2 m V(\vec{r})\right) \phi_{\eta_{c}}(\vec{r})=\mathcal{E}^{2} \phi_{\eta_{c}}(\vec{r})
$$

where $m$ is the reduced mass of the $\eta_{c}$-nucleus system in vacuum and $V(\vec{r})$ is the $\eta_{c}$-nucleus potential given in Eq. (7).

The computed bound state energies $(E)$ of the $\eta_{c}$-nucleus system, given by $E=\mathcal{E}-m$, where $\mathcal{E}$ is the energy eigenvalue in Eq. (8), are listed in Table 1 for four values of $\Lambda_{D}$. The results in Table 1 show that the $\eta_{c}$-meson is expected to form bound states with all the nuclei considered, independently of the value of $\Lambda_{D}$. Clearly, however, particular values for the bound state energies are dependent on $\Lambda_{D}$. This dependence was expected since the $\eta_{c}$ potentials are also dependent on $\Lambda_{D}$, and it is therefore an uncertainty in the results obtained in our approach. Finally, note that the binding is stronger for larger values of $\Lambda_{D}$ and that the $\eta_{c}$ is predicted to be bound more strongly to heavier nuclei.

\section{Summary and Conclusions}

We have calculated the $\eta_{c}$-nucleus bound states energies for various nuclei. The $\eta_{c}$-nucleus potentials were calculated in the a local density approximation from the $\eta_{c}$ mass shift as a function of the nuclear matter density and the baryon density profiles of the nuclei studied. Using these potentials, we have solved the Klein-Gordon equation to obtain $\eta_{c}$-nucleus bound state energies. Our results show that one should expect the $\eta_{c}$ to form bound states for all the nuclei studied, even though the precise values of the bound state energies are dependent on the cutoff mass values 
$\Lambda_{D}$ used in the form factors. The discovery of such bound states would represent an important step forward in our understanding of the nature of strongly interacting systems. Note that we have ignored the natural width of $32 \mathrm{MeV}$ in free space of the $\eta_{c}$ and this could be an issue related to the observability of the predicted bound states. However, it should still be possible to see that there are bound states, which is the main result of this work. Addition of an imaginary part to the $\eta_{c}$ potentials is underway and will be reported elsewhere. Furthermore, we have done an inital study [6] using a different form factor since this may impact the results reported here.

\section{Acknowledgments}

This work was partially supported by Conselho Nacional de Desenvolvimento Científico e Tecnológico (CNPq), process Nos. 313063/2018-4 (KT), 426150/2018-0 (KT) and 309262/2019-4 (GK), and Fundação de Amparo à Pesquisa do Estado de São Paulo (FAPESP) process Nos. 2019/007630 (KT), 64898/2014-5 (KT) and 2013/01907-0 (GK). The work is also part of the project Instituto Nacional de Ciência e Tecnologia - Nuclear Physics and Applications (INCT-FNA), process No. 464898/2014-5 (KT, GK). It was also supported by the Australian Research Council through DP180100497 (AWT).

\section{References}

[1] S. J. Brodsky, I. Schmidt and G. de Teramond, Phys. Rev. Lett. 64 (1990), 1011

[2] G. Krein, A. W. Thomas and K. Tsushima, Prog. Part. Nucl. Phys. 100, 161 (2018)

[3] G. Krein, A. W. Thomas and K. Tsushima, Phys. Lett. B 697 (2011), 136-141

[4] K. Tsushima, D. Lu, G. Krein and A. W. Thomas, Phys. Rev. C 83 (2011), 065208

[5] J. J. Cobos-Martínez, K. Tsushima, G. Krein and A. W. Thomas, Phys. Lett. B 811 (2020), 135882

[6] G. N. Zeminiani, J. J. Cobos-Martinez and K. Tsushima, Eur. Phys. J. A 57 (2021) no.8, 259

[7] J. J. Cobos-Martínez, K. Tsushima, G. Krein and A. W. Thomas, Phys. Lett. B 771 (2017), $113-118$

[8] J. J. Cobos-Martínez, K. Tsushima, G. Krein and A. W. Thomas, Phys. Rev. C 96 (2017) no.3, 035201

[9] Z. w. Lin and C. M. Ko, Phys. Rev. C 62, 034903 (2000)

[10] W. Lucha, D. Melikhov, H. Sazdjian and S. Simula, Phys. Rev. D 93, no. 1, 016004 (2016); Addendum: [Phys. Rev. D 93, no. 1, 019902 (2016)]

[11] K. Saito, K. Tsushima and A. W. Thomas, Phys. Rev. C 56, 566 (1997) 